\title{
Analysis on Evolution of Style of Chinese Classical Dance from Classical Dance Play
}

\author{
Xiaoya Long \\ School of music and dance, Jishou University, Jishou Hunan, 416000, China
}

Keywords: Classical dance play, Chinese classical dance, Style evolution.

\begin{abstract}
Through long-time development, the dance type - Chinese classical dance has made certain achievements, formed a distinct dance style and enriched Chinese dance art. Starting with connotations and artistic characteristics of Chinese classical dance, this paper analyzes the evolution of the style of Chinese classical dance with classical dance play as entry point and expects to further enrich theoretical guidance of artistic style of Chinese classical dance and promote better and more farsighted development of Chinese classical dance art in the future.
\end{abstract}

\section{Introduction}

Dance is a body art form spreading for the longest time in each country. Dance forms considered as national dance model in different historical periods in China have a greater value in the research on Chinese dance art. Modern and contemporary dance researchers and performers define dance with classical style created in modern times and traditional classical dance forms as Chinese classical dance uniformly in the process of research and practice, and point out that this dance form pursues for "the integration of body and sign" in the process of performance and is one of the representatives of traditional Chinese culture. This shows not only the inheritance of traditional excellent Chinese historical culture by the current society, but also the integrative development trend of traditional culture and modern culture in the current society. It has corresponding positive influence on good inheritance of Chinese classical dance. However, with social development and the invasion of foreign culture and when the aesthetic standard of the public constantly changes, the overall style of Chinese classical dance is under constant exploration under the influence of communication subject. This paper attempts to conduct a specific analysis on style evolution of Chinese classical dance based on its concept and artistic characteristics.

\section{Concept of Chinese classical dance}

Since 1950s, Chinese classical dance has been named formally. In spite of this, the definition of its concept has been fuzzy for a long time under the influence of dislocation and loss of historical times. After Chinese classical dance was named, the concept of classical dance has been argued in the academic world. Several uniform opinions have formed up to now, i.e. contemporary Chinese classical dance, post classical dance and new classical dance ${ }^{[2]}$.In the analysis from the perspective of genetic epistemology, it should be noted that a tectonic form that has not been defined clearly exists in the study on the concept of Chinese classical dance. Therefore, to comprehensively understand and analyze the reason of generation and operating mechanism of such construct, it is required to explore multiple development stages it has experienced. Strictly speaking, Chinese classical dance refers to dance considered as official dance model in each historical period in China and dance with certain classical style created by people in the modern society in general. This concept has certain implication and contains meanings at two levels: first, dance forms considered as official model in each historical period in ancient China; second, dance forms with ancient customs and charm created by contemporary people under the influence of contemporary artistic thought. That is to say, one meaning is ancient dance and another form is classical dance. They have certain connection and differences. It is thus clear that the precondition of determining connotations of Chinese classical dance is that classical dance can be ancient typical dance but is not equal to ancient dance; it is not 
dance in a specific historical period, but should be classical dance form with ancient customs and charm that can be created according to the development of era. Therefore, it is required to enhance the importance attached to ancient dance and modern dance with ancient customs in two aspects in the process of studying Chinese classical dance. Only with comprehensive analysis on connotations of Chinese classical dance can we explore its style evolution in its development history based on its connotations and lay a solid foundation for the research on Chinese classical dance art.

\section{Artistic characteristics of Chinese classical dance}

\section{Opera dance is the predecessor of Chinese classical dance.}

At the beginning of the foundation of New China, Chinese society experienced a hot trend of learning traditional classical culture and art with the establishment of the state and the gradual improvement of a sense of national pride of the public in social development. Especially after the Chinese nation started to stand firm among the nations of the world independently, Chinese national culture must integrate the tradition, get rid of the stale and bring forth the fresh, develop its personalized art form and then impel Chinese art to stay in the world art with vigorous life and achieve innovation and inheritance in order to achieve better development in the world. For Chinese classical dance, the most representative "body charm classical dance school” of Tang Mancheng considers opera dance as the predecessor of classical dance. Therefore, it analyzes artistic characteristics of Chinese classical dance, guides the study on Chinese classical dance with systematic study on opera dance under the premise of the study on opera dance and promotes better good inheritance and development of Chinese classical dance. As famous Chinese classical dance researcher Ouyang Yuqian said in his writing Yi De Yu Chao, "Chinese classical dance has been preserved well in opera dance. Its specific performance techniques can have certain reference and guidance functions for modern Chinese classical dance performance.” This reflects that opera dance contains the essence of ancient Chinese dance in historical inheritance to a certain extent. In particular, the content and form of classical dance are inherited well in the essence of Chinese opera art Peking Opera and Kun Opera, which have significant influence on the development of Chinese classical dance. Meanwhile, traditional Chinese opera art has certain beauty of body, charm and image and high accuracy and can meet the demand of aesthetic pattern of Chinese classical dance in the development. Therefore, requirements of era development and characteristics of art noumenon impel Chinese opera dance to be the predecessor of Chinese classical dance and produce certain impellent influence on the development of Chinese classical dance.

\section{Body charm of Chinese classical dance.}

In the field of Chinese dance art, Chinese classical dance gathers the essence of traditional Chinese dance art and reflects the aesthetic style of the whole nation to a certain extent. It is not ancient dance in the strict sense and has certain differences from opera dance. However, it obviously contains dance charm of the Chinese nation handed down from ancestors. Chinese classical dance extracts personalized charm in the development process. Due to the practice of body charm in the process of performance, performers impel Chinese classical dance performance to change from external formal imitation to the mastery of internal charm and endow classical dance with a soul at spiritual level. For Chinese classical dance, the main feature of body charm is that it removes requirements for body movement in the process of performance, combines body and rhythm perfectly and finally achieves an artistic effect of "integrating body and charm”. In dance performance, body charm often considers "body, spirit, vigor and rhythm" as the basic starting point and final home of modeling. "Body" refers to all external patterns of manifestation of dance movements; "spirit" refers to charm, i.e. thought and artistic conception to be expressed by dance performers; "vigor" refers to internal rhythm and the change of dynamics of dance, e.g. speed and force in dance; "rhythm" is specifically manifested as internal and external law of motion of dance movements.

In the performance of Chinese classical dance, body rhyme starts with relevant constitution elements of dance movements and explores the source in the actual performance process of performers. Each movement is closely related to the thought and breath of dancers. Therefore, breath 
training of performers in dance training can improve the sense of extension and inner sense of their dance performance ${ }^{[3]}$. "The beauty of form touches eyes and the beauty of meaning touches the heart" reflects the spiritual style and aesthetic connotation of Chinese classical dance. Rich dancing posture, certain national style and strong expressive force have gradually developed into the core identification of Chinese classical dance. They are art spirit of the inheritance and development of Chinese classical dance in the modern society. They have certain influence on style evolution of classical dance in different historical periods and certain research value.

\section{View on style evolution of Chinese classical dance from classical dance play}

The formation of body rhyme and movement of Chinese classical dance connects its unique Chinese cultural connotations and inherent spirit with aesthetic tendency in contemporary Chinese cultural art organically, more emphasizes "the guidance over body with spirit" in terms of body rhyme manifestation, focuses on "breath" in the performance of dance art and judges the artistic value of dance performance with the control over "breath". That is to say, the correct use of breath has gradually become the lifeblood of Chinese classical dance performance in the development process of classical dance. Let' $s$ take the analysis on Artificial Identity for example. We can see the breath use mode of the dancer in the movement process at the beginning of the performance. Each movement is integrated with the breath of the dancer perfectly, thus greatly improving the artistic value of dance performance. Therefore, "breath" has started to become an important standard for checking the harmony of dance movements of dancers and perfect deduction of spiritual connotations of dance in the field of modern dance performance art. Great importance has been attached to it by dance performers and researchers.

With the development of classical dance, in Ode to the Lotus Flower, dancers pay more attention to poetic dance structure in the process of performance and fade their emotional expression. However, typical features with flexibility, beauty in appearance and intelligence are endowed to the female image in the poem. It can be seen that choreographists in the play have started to use modern choreography technique to create dance movements of classical dance or capture a specific aesthetic thought of classical dance in action deduction for appropriate adjustment, thus impelling the whole dance performance to have rich classical spirit and distinct modern spirit of innovation and creating conditions for the inheritance of classical dance in the modern society ${ }^{[4]}$.In creative practice, cainozoic choreographists comprehensively master root-taking system, break the tradition, keep a foothold in the contemporary times, seek for innovative spirit and thought on this basis, integrate the essence of modern dance art appropriately in the creation process of classical dance and open a new path for the development of Chinese classical dance to a certain extent.

Therefore, in terms of the analysis on classical dance play, traditional classical dance plays attach more importance to body movement, perfect combination between breath and dance movement in actual performance and the increase of artistic connotations of dance in "body, spirit, vigor and rhythm”. Classical dance works have high artistic value ${ }^{[5]}$.However, developing up to the current society, Chinese classical dance has gradually entered the historical period of transitional development. Under the social background of economic globalization, same as other multiple forms of art, dance art is faced with conflicts and dilemmas between tradition and modern, locality and globality and perseverance and westernization in the inheritance process. Meanwhile, social transitional development causes certain changes of aesthetic idea of the public. Such aesthetic changes produce certain influence on the change of body movement and rhyme of classical dance, impel choreographists of classical dance to endow specific style and flavor to movement elements more carefully, e.g. Kong Yiji, Soratani Orchids and Rouge, and constitute the new style trend of classical dance in the modern society.

With historical changes and cultural accumulation over thousands of years, at the beginning of formation of Chinese classical dance, its rhyme has laid the development keynote of Chinese classical dance performance which respects classical aesthetics in the development. In the harmony of body and spirit, it is required to "guide body with spirit and convey spirit with body". Only in this way can 
classical dance stick to the keynote thought in the transitional development process of each stage, maintain its nature in the inheritance over thousands of years and manifest the connotation of traditional Chinese culture constantly. For example, classical traditional plays The Butterfly Lovers and Mulan Ballad etc. are typical standard models of classical dance. Their dancers use "spirit" as the source of manifesting expressive force of dance art in performance. Even with repeated creation, they still have strong artistic appeal and can reflect the essence of traditional Chinese cultural art. In the contemporary society, the action form of classical dance has changed under the influence of modern choreography skills. On the basis of sticking to body rhyme of Chinese classical dance, modern choreographists have made appropriate adjustments to the action and performance form of classical dance. They not only realize the innovation of classical dance, but also endow new connotations of classical dance plays. Aesthetic demands of appreciators in the modern society can be met. For example, in Orange Red, Book Rhyme and When Will You Come Back, the creative thought of Chinese classical dance and the pattern of manifestation of modern social dance are combined in their choreography. The pursuit for classical dance and art in modern dance development is reflected in the performance. These dance forms not only have rich classical implications, but also manifest distinct modern choreography forms and artistic movements and realize perfect combination of spirit and body. Only with the combination of external movement and overall style in the process of creation and performance can we create Chinese classical dance works with typical features and classical style and flavor, produce certain positive influence on further promoting the inheritance and development of Chinese classical dance in modern society and facilitate the progress of Chinese dance art.

\section{Conclusion}

In conclusion, Chinese classical dance art plays a vital role in the field of traditional Chinese cultural art. Its good development path has corresponding positive influence on the improvement of influence of the overall Chinese cultural art. Therefore, it is required to attach more importance to the inheritance and development of Chinese classical dance in the new period, promote the integration of classical dance on the basis of sticking to the essence of Chinese classical dance, pay attention to features of style and flavor of works, promote the innovation and development of Chinese classical dance and make Chinese classical dance inherited better in the future and play a positive role in the inheritance of Chinese cultural art and the improvement of world appeal of Chinese culture.

\section{Acknowledgments}

This paper is course teaching reform program of Jishou University in 2015.

\section{References}

[1] Jin Hao. View on Ten Years of Development of Chinese Classical Dance in New Century. Shanghai: Shanghai Music Publishing House, 2011 (12).

[2] Lv Bi. Discussions on Training and Application of Techniques and Skills of Chinese Classical Dance, Home of Opera, 2015(11):123-124.

[3] Liu Zhiya. As for the Inexhaustible Source - Analysis on Dance Forms and Image Experience of Classical Play Ta Ge, Home of Opera, 2015(9):147-147.

[4] Ma Jun. View on Virile Style of Wushu Dance Performance in Chinese Classical Dance from Work Zhan Gu Xing, Journal of Beijing Dance Academy, 2014(z2):90-92.

[5] Zhao Jing. Style and Personalized Creation of Chinese Classical Dance, Journal of Shenyang Normal University: Social Science Edition, 2012,36(4):151-152.

[6] Lei Bingqi. Discussions on Style Evolution of Chinese Classical Dance from Classical Dance Plays, Exam Weekly, 2013(99):25-26. 